\title{
On-Demand Droplet Merging with an AC Electric Field for Multi- ple-Volume Droplet Generation
}

\author{
Adrian J.T. Teo, Say Hwa Tan, and Nam-Trung Nguyen* \\ Queensland Micro- and Nanotechnology Centre, Griffith University, 170 Kessels Road QLD 4111, Brisbane, Australia.
}

\begin{abstract}
We introduce a unique system to achieve on-demand droplet merging and splitting using a perpendicular AC electric field. The working mechanism involves a micropillar to split droplets, followed by electrocoalescence using an AC electric field. Adjusting the parameters of the AC signal and conductivity of the fluid result in different merging regimes. We observed a minimum threshold voltage and a strong influence of the surfactant. We hypothesize that the merging process is caused by dipole-dipole coalescence between the daughter droplets. At the same time, adjustment of the conductivity reveals a shift in the merging regimes and can be explained with an electric circuit diagram. Size-based sorting using this merging phenomenon is subsequently demonstrated, where alternate, single, double and triple droplets sorting were achieved. The concept presented in this paper is potentially useful for drug dispensing or multi-volume digital polymerase chain reaction, as droplets of multiple sizes can be generated simultaneously.
\end{abstract}

Droplet coalescence is a process where two or more droplets come together to form a single larger droplet. ${ }^{2}$ Being one of the manipulation tasks in droplet-based microfluidics, it is useful for mixing controlled amounts of different fluids in a contaminant-free environment. Controlled coalescence and dispensing of droplets have a broad range of applications such as protein engineering ${ }^{3}$, drug screening ${ }^{4}$ and oil refining. ${ }^{5}$ Droplet coalescence has been widely studied ${ }^{6-8}$ and is achievable using either passive or active methods. Passive methods utilize fixed structures such as pillar microarrays ${ }^{9}$ or expansion channels ${ }^{10}$ to manipulate the flow characteristics of the droplets ${ }^{7}$. Active methods involve external energy sources to disrupt the film bridge or mechanically speed up the draining process of the film between the droplets. ${ }^{11}$ Active methods provide additional benefits such as selectivity and on-demand capabilities, correspondingly saving experimental resources. Some examples of these are pneumatic, ${ }^{12}$ acoustic ${ }^{13}$ and electric field. ${ }^{14,15}$ Droplet handling using electric fields is one of the most commonly used methods as its influence and positioning within a device is highly adjustable. It also does not cause any biocompatibility issues when used within an appropriate range or affect the droplet medium itself.

Utilizing electric fields in droplet merging, also known as electrocoalescence, have been comprehensively studied. ${ }^{16,17}$ If the droplets are charged in a uniform field, droplets move towards each other due to electrophoresis induced by the electric field and the double layer around the droplet. ${ }^{17-20}$ In the presence of an electric field, coalescence subsequently occurs through destabilization of the droplet-droplet interface. ${ }^{11}$ In the presence of a uniform electric field, neutral droplets undergo deformation. ${ }^{21}$ The droplet is accordingly stretched in the same direction as the electric field due to the induced electric Maxwell stresses on the surface, ${ }^{22}$ with Taylor cones formed under high electric field strength. ${ }^{23}$ If the droplets flow in parallel to the uniform field, an attractive force results from the dipole-dipole interaction. ${ }^{24}$ This force brings the droplets closer, increasing in strength as the distance between the droplets decreases. ${ }^{15}$ Subsequently, film thinning occurs where the rate of thinning can be tuned by the applied electric field. ${ }^{25}$ In a non-uniform electric field, coalescence may take place through dielectrophoresis. ${ }^{26}$ Surfactants are also commonly used during experiments to prevent unexpected merging of droplets. The activation of the electric field would cause these surfactant molecules to redistribute and realign under electric field. ${ }^{27,28}$ 
The influence of the electric field on the droplets is highly complex, thus electrocoalescence studies were usually carried out under multiple constrains. Most studies are based on an electric field parallel to the flow direction of droplets, ${ }^{15,29-31}$ whereas others require the assumption that the droplets are spherical outside the electric field. Another limitation is that droplets also need to be approximately close to each other, leading to a need for synchronization of droplets in the merging chamber. ${ }^{15}$ Accordingly, these limitations require an ideal setup for electrocoalescence, however this is unrealistic as not all droplets flow along the same direction as the electric field, nor are they always spherical. Because of these limitations, a knowledge gap still remains for understanding droplet merging in microfluidics. The present paper introduces a coalescence concept with an AC electric field that is perpendicular to the flow direction of the two daughter droplets. We made use of a 30 $\mu \mathrm{m}$ micropillar placed in the middle of a microchannel to prepare the daughter droplets, Figure 1. In previous works, micropillars were used to slow down the droplets. ${ }^{12,30}$ However, the pillar in our device acts as an obstacle to split the droplet, such that the two daughter droplets are positioned alongside of each other ${ }^{32,33}$. This arrangement accordingly allows for positioning of the daughter droplets parallel to the electric field, without affecting their flow direction. It also effectively eliminates the need for synchronization required in all other works reported to date in the literature. Activating an AC electric field perpendicular to the flow joins the two daughter droplets. Varying the applied voltage $V_{A P P}$, the frequency $f$ and the conductivity of the dispersed phase $k$ leads to three merging regimes: breakup, merging after pillar and merging at pillar. With insights into the well-controlled merging regimes, we subsequently demonstrated size-based sorting using an expansion channel. Size-based sorting eliminates the need for subsequent manual sorting of droplets during analysis. ${ }^{34}$ It also enables more efficient analysis for concentration varying experiments. ${ }^{35}$ We envisage that this system would have potential applications in droplet dispensing and controlled generation of multi-volume droplets.

\section{MATERIALS AND METHODS}

(a)

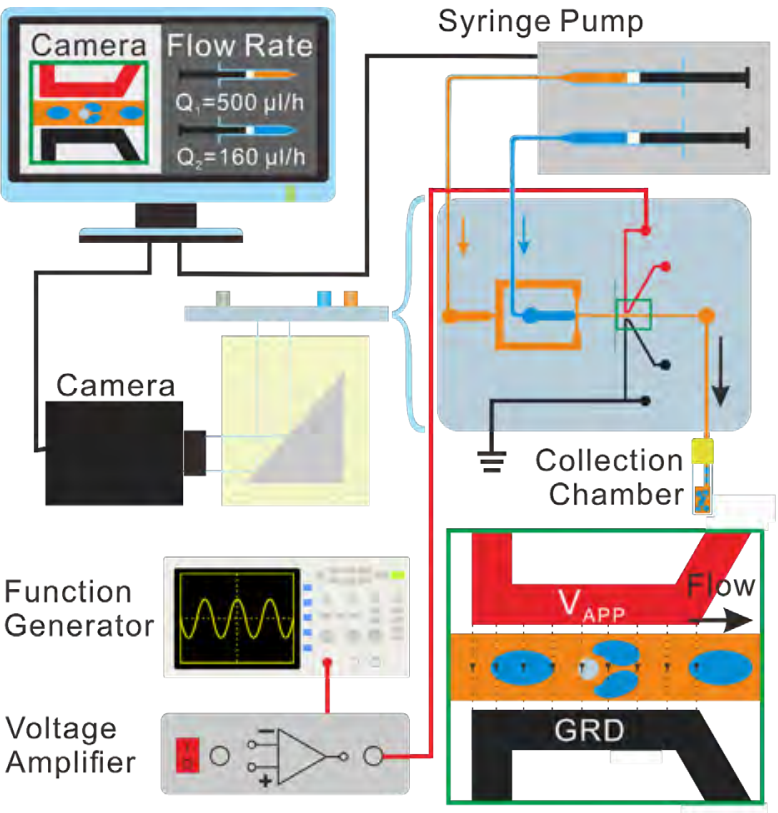

(b)

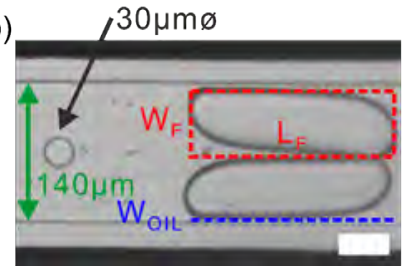

(c)

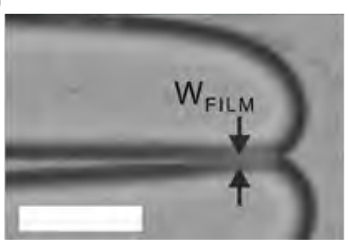

Figure 1. On-demand droplet merging and splitting: (a) Schematic diagram of experimental setup illustrating droplet merging upon activation of AC electric field generated across the microchannel by electrodes (inset). (b) Dimensions used for the electric circuit model. (c) Captured images of the oil film between the daughter droplets. Scale bars depict $50 \mu \mathrm{m}$.

A microfluidic device made of polydimethylsiloxane (PDMS) with a flow focusing configuration was developed as given in Figure 1a. The device was fabricated using standard photo and soft lithography methods. ${ }^{36}$ The unique device consists of a $30 \mu \mathrm{m}$ round PDMS pillar positioned in the middle of a $140 \mu \mathrm{m}$ wide main fluidic channel to break up passing droplets, Figure $1 \mathrm{~b}$. This pillar is also placed between 
two electrodes that generate the electric field for droplet merging. These electrodes are fabricated using the same mold as the fluidic channel to eliminate the need for precise alignment of microchannels and electrodes. ${ }^{37,38}$ Both the electrodes and microchannels have the same height of $29 \mu \mathrm{m}$.

A schematic diagram of the experimental setup is given in Figure 1a. A syringe pump (neMESYS, Centoni GmbH) delivered the fluids into the device with the flow rates controlled from the computer. Across the main microchannel, one electrode (red) was connected to a function generator (33210A, Keysight Technologies) through a voltage amplifier (Model 623B, Trek Inc). The other electrode (black) was connected to ground. An oscilloscope (TBS1102B, Tektronix) displayed and recorded the values for the applied AC voltage $V_{A P P}$ and its frequency $f$. The device was placed on an inverted microscope (Eclipse Ti, Nikon Instruments), and videos were recorded using a highspeed camera (Phantom Miro3, Vision Research) connected to the same computer.

The continuous phase used was mineral oil (M5904 with 1 wt \% SPAN80, Sigma Aldrich). The dispersed phase used was a series of salt solutions of a different conductivities $k$. This was obtained by adding sodium chloride (NaCl, Chem-Supply Pty Ltd) to deionised (DI) water. The interfacial tension between the fluids was measured as $5.5 \mathrm{mN} / \mathrm{m}$ (Sinterface, PAT1). The dynamic viscosity of the oil and salt solutions were $23.8 \mathrm{mPa} . \mathrm{s}$ and $1 \mathrm{mPa}$.s, resepectively. ${ }^{39}$ Throughout the experiments, the standard flow rates were $Q_{O}=500 \mu \mathrm{l} / \mathrm{h}$ for the continuous phase and $Q_{w}=160 \mu \mathrm{l} / \mathrm{h}$ for the dispersed phase unless otherwise mentioned. At these flow rates, the average droplet area was measured as $23,000 \mu \mathrm{m}^{2}$.

\section{RESULTS AND DISCUSSION}

Experiments were carried out to investigate the effect of the AC frequency $f$ and the magnitude $V_{A P P}$ of the applied voltage. Preliminary experiments were done to observe if merging would occur in small and large droplets. The results are provided in Supporting Information S1, showing that droplets were successfully merged at all sizes. Subsequently, we focus on the generation of larger droplets and tuned the frequency $f$ from $1 \mathrm{kHz}$ to $90 \mathrm{kHz}$ and the applied voltage $V_{A P P}$ from $10 V_{R M S}$ to $180 V_{R M S}$, Figure 2a. The videos were captured at 1 ,200 frames/sec. Figure 2b shows the representative images of the merging regimes observed in our experiments. Before activating the electric field, the droplets remain separated as shown in the "No AC Field” inset of Figure 2b. At low frequency and voltage, no merging was observed, as represented by the triangles in Figure 2a and the "Breakup” inset. As the voltage gradually increased, the first instance of merging was observed, Figure 2a. This transition line between "Breakup” and "Merge after Pillar” is where inconsistent merging (“Inconsistent Merging” inset) was observed and labelled by the circle markers. The inset in Figure 2b shows two different instances of without merging (left inset) and with merging (right inset). Increasing the voltage past this point led to consistent merging ("Merge after Pillar” inset) as depicted by the square markers. The merging phenomenon happens after the droplet exits the pillar. As the applied voltage further increased, another inconsistent regime was observed, where occasionally merging happened right at the pillar ("Inconsistent Merging at Pillar" inset). This regime is depicted with inverted triangle markers, where the droplet merged even before fully exiting the pillar (right inset). With increasing frequency, we observed that all droplets merged at the pillar (“Merge at Pillar” inset), labelled as diamond markers. Supporting Information S2 provides representative videos of the different regimes.

According to recorded videos, each droplet was observed to traverse pass the electrode region for a duration of 25 milliseconds for the given fixed flow rates. The droplet enters the electrode region for approximately 10 milliseconds before encountering the micropillar. The droplet subsequently takes approximately 5 milliseconds to completely pass through the pillar and another 10 milliseconds to fully exit the electrode region. The charge relaxation times for the series of conductivities used are in the range of $\tau=10^{-10} \sim 10^{-6}$ seconds according to the equation $\tau=\varepsilon_{0} \varepsilon_{r} / k$. Charge relaxation time is defined as the time taken for the distribution of charge within a medium to reach an equilibrium. ${ }^{40}$ The AC signal is introduced using a sine wave with frequencies from $1 \mathrm{kHz}$ to $100 \mathrm{kHz}$, corresponding to a period of

$10^{-5} \sim 10^{-3}$ seconds, much larger than the charge relaxation time. Thus, the fluids used in our experiments are able to distribute the charges faster than the excitation frequency of the field. Concurrently, the charges are able to redistribute during the splitting of the droplet such that the daughter droplets remain neutral. This timescale analysis effectively rules out electrophoresis as the contributing factor for this merging phenomenon as the droplets are not charged. The field-induced dynamic instability described by Priest et. al. ${ }^{41}$ is also not applicable for our experiment as their droplets are observed to be charged according to the above timescale analysis.

Another mechanism that could explain the merging phenomenon is dielectrophoresis ${ }^{11}$. However this phenomenon involves a non-uniform electric field, where the droplet would shift towards the region of maximum field gradient. ${ }^{17}$ This is more commonly used as an actuation 

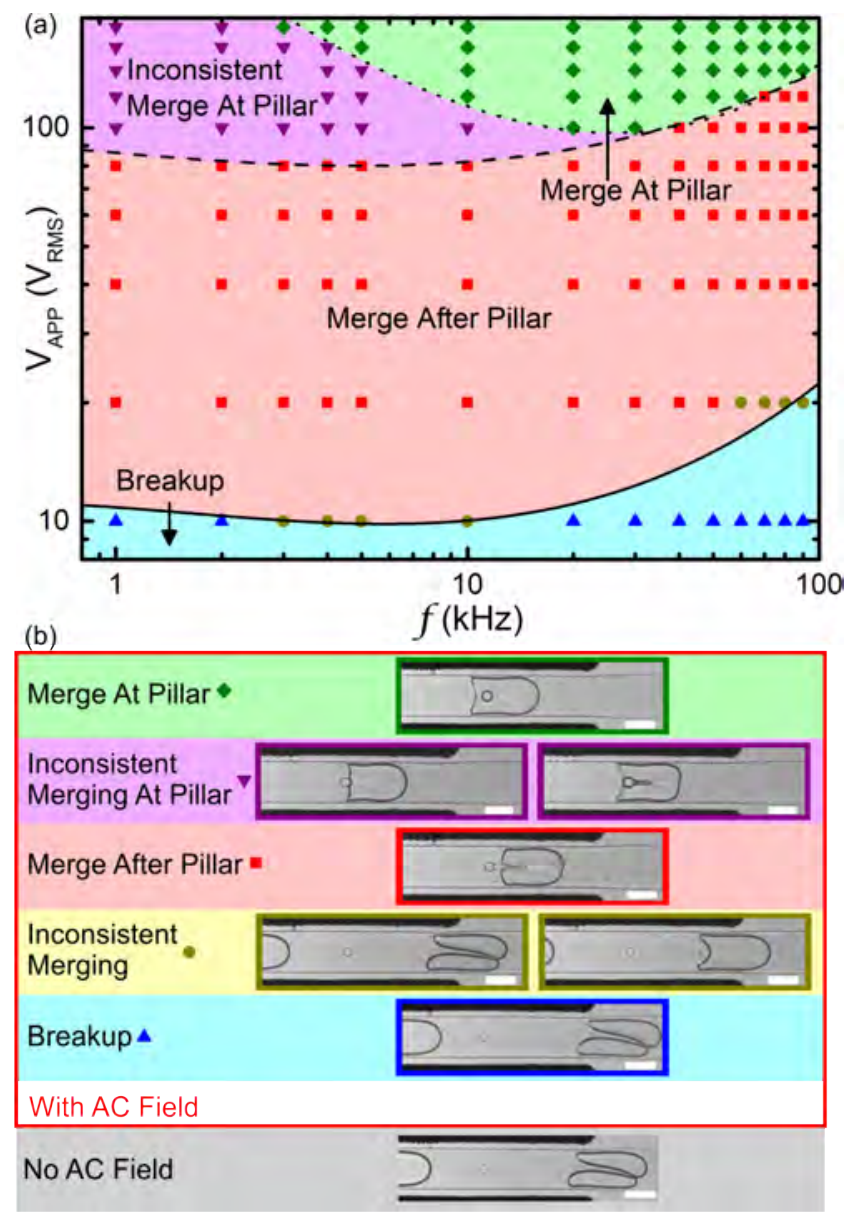

Figure 2. Merging regimes: (a) Operation map for a solution of $0.76 \mu \mathrm{S} / \mathrm{cm}$ showing different stages of droplet merging with lines depicting the boundaries. (b) Representative images of the different merging regimes. Merged droplet insets show the first frame upon merging. Scale bars represent $100 \mu \mathrm{m}$.

mechanism for on-demand droplet sorting . ${ }^{42,43}$ In our experiment, we used a uniform electric field instead with the use of the $750 \mu \mathrm{m}$ long electrodes parallel to the main fluidic microchannel. Influences of the PDMS micropillar on the electric field are also negligible as observed in the experiment videos. Therefore, it is logical to eliminate this option, leaving the last mechanism of dipole-dipole coalescence.

We previously stated that the droplets remain uncharged even after splitting. Also, after splitting and exiting the pillar, the droplets were still within the uniform electric field for at least 10 milliseconds. This is observed by recording the frame where the droplets exit the parallel electrode region. During this period, the surface charge of both droplets is constantly modulated by the AC signal, because the charge relaxation time of the droplets is faster than the AC voltage. Accordingly, this results in two different mechanisms for coalescence. Firstly, the induced Maxwell stresses upon each droplet surface results in a deformation of the droplet shape. This deformation subsequently squeezes the thin film of oil which speeds up the coalescence. Nevertheless, Figure 1c reveals that the width of the oil film is relatively small, reducing the potential effect of the squeezing phenomenon. A second phenomenon for coalescence that has a greater influence is the polarization of the surface charge of each daughter droplet. With the increasing electric field strength $E=V_{A P P} / d$ where $d$ is the channel width, we observed that the merging phenomenon also increases in intensity with "Merging After Pillar” transiting to "Merging At Pillar".

The regime map in Figure 2a reveals a minimum threshold voltage required for merging. The threshold is indicated as the line between “Breakup” and "Merge After Pillar”. This voltage level was observed to slightly increase with increasing frequency $f$. First, we attribute this to the surfactant in the continuous phase. Initial experiments carried out without SPAN80 showed droplet merging even without the AC field. The same observation was reported by Niu et. al. ${ }^{30}$ for higher electric field strength and higher concentrations of SPAN80 in hexadecane. The concentration of SPAN80 used in our experiments was kept near the critical micelle concentration (CMC) level ${ }^{44}$ for stable droplet formation during splitting of the droplets. The surfactant molecules therefore attach to the droplet surface, while concurrently forming reverse 
micelles. ${ }^{45}$ Under the electric field, these molecules realign and redistribute themselves resulting in merging. This phenomenon has been previously reported ${ }^{27,28}$ and is therefore outside the scope of this paper.

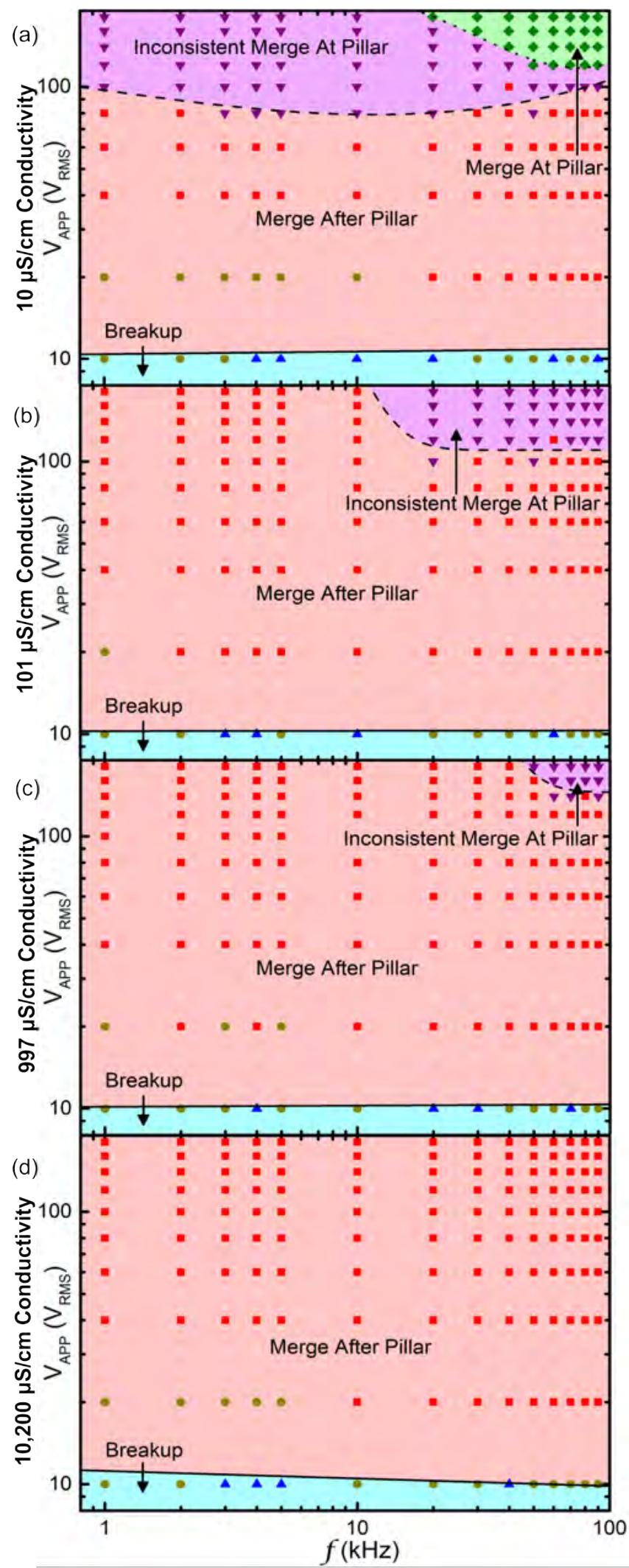

Figure 3. Regime maps for different conductivities (a) $10 \mu \mathrm{S} / \mathrm{cm}$ (b) $101 \mu \mathrm{S} / \mathrm{cm}$ (c) $997 \mu \mathrm{S} / \mathrm{cm}$ and (d) $10,200 \mu \mathrm{S} / \mathrm{cm}$ showing consistent transition voltage for start of merging. Regimes for consistent and inconsistent merging at pillar however are observed to shift away from observed frequency and voltage range at higher conductivities. 
We repeat the same experiment with a series of salt solutions as the dispersed phase, where $k=10 \mu \mathrm{S} / \mathrm{cm}, 101 \mu \mathrm{S} / \mathrm{cm}, 997 \mu \mathrm{S} / \mathrm{cm}$ and 10,200 $\mathrm{SS} / \mathrm{cm}$. Figure 3 shows the regimes obtained for each conductivity. The threshold voltage remained the same throughout the different conductivities. This further confirms our earlier hypothesis that the field mainly affected the surfactant in the continuous phase. Furthermore, as the conductivity increased, the inverted triangle and diamond markers shift to the top right corner of the voltage-frequency space. Merging at pillar regime subsequently occurred at a conductivity of $10,200 \mu \mathrm{S} / \mathrm{cm}$, indicating that the intensity of the electric field was greatly reduced. This intensity is correlated with the voltage across the daughter droplets, which can be theoretically estimated with an electric circuit diagram shown in Figure 4. Further details of each electrical component are given in the corresponding schematic diagram. The ratio between the voltage across each daughter droplet and the excitation voltage is given as

$$
\frac{V_{F}}{V_{A P P}}=\left(2+\frac{2}{A C_{P D M S}}+\frac{2}{A C_{O I L}}+\frac{1}{A C_{F I L M}}\right)^{-1}
$$

where $V_{F}$ is the voltage across the daughter droplets, $A=j \omega Z_{F}$, with $j^{2}=-1, \omega=2 \pi f$ and $Z_{F}$ is the impedance of the droplet given by $Z_{F}=\frac{L_{F}}{k W_{F} H}$. Here, $L_{F}$ refers to the droplet length, $W_{F}$ is the average width of the daughter droplet and $H$ the height of the channel. $C_{P D M S}$ is the capacitance of the PDMS wall, where $C_{P D M S}=\varepsilon_{o} \varepsilon_{r P D M S}\left(\frac{L H}{W_{P D M S}}\right), \varepsilon_{o}$ refers to the vacuum permittivity of $8.85 \times 10^{-12} \mathrm{~F} / \mathrm{m}, \varepsilon_{r P D M S}$ the relative permittivity of 2.5 for PDMS, $L$ the length of the electrode, $W_{P D M S}$ the width of the PDMS wall of $23 \mu \mathrm{m}$. $C_{O I L}$ is the capacitance of the continuous phase near the wall with $C_{O I L}=\varepsilon_{o} \varepsilon_{\text {rOIL }}\left(\frac{L_{F} H}{W_{O I L}}\right), \varepsilon_{\text {rOIL }}$ the relative permittivity of 2.5 for the continuous phase ${ }^{46}$ and $W_{O I L}$ the width of the oil film experimentally measured to be approximately $4 \mu \mathrm{m}$. The different dimensions are given in Figure $1 \mathrm{~b}$. Lastly, $C_{F I L M}$ is the capacitance of the thin film of oil that separates the two daughter droplets, where $C_{F I L M}=\varepsilon_{o} \varepsilon_{r O I L}\left(\frac{L_{F} H}{W_{F I L M}}\right)$, and $W_{F I L M}$ is experimentally measured to be approximately $1 \mu \mathrm{m}$. For the calculation of the electric circuit, we provide a broad approximation where each daughter droplet is a rectangular cuboid of volume $L_{F} \times W_{F} \times H$. Using this equation, we plot $\frac{V_{F}}{V_{A P P}}$ against $f$ as given in Figure 4a.
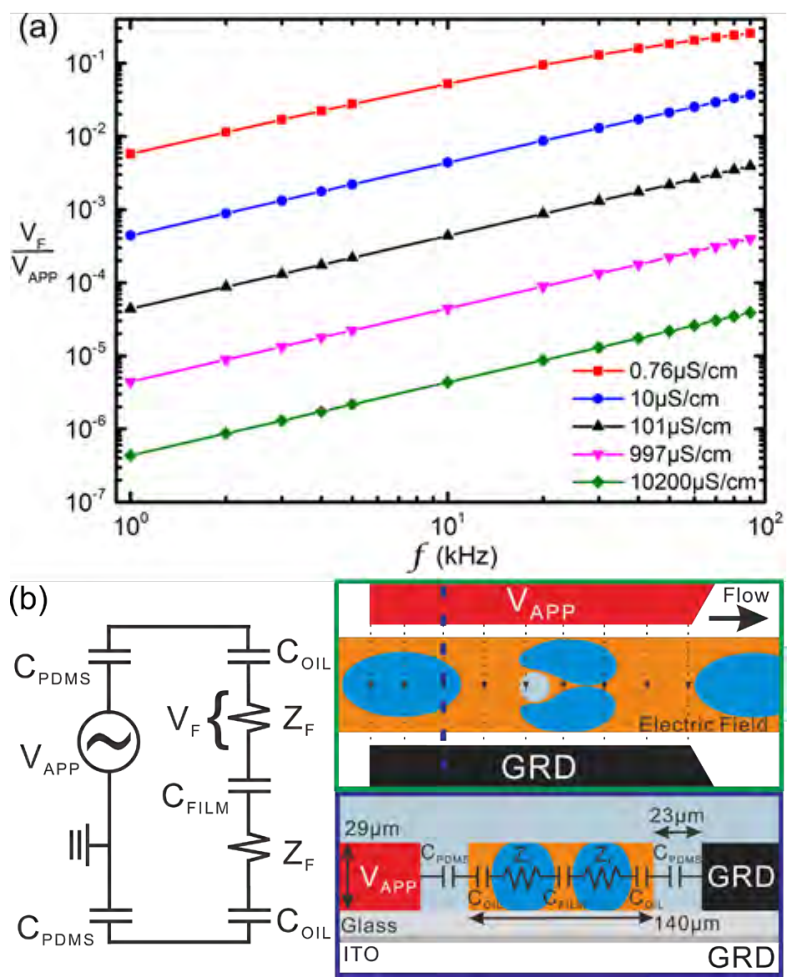

Figure 4. Electric circuit of the setup: (a) Theoretical evaluation of $\mathrm{V}_{\mathrm{F}} / \mathrm{V}_{\mathrm{APP}}$ against $\mathrm{f}$ across a series of dispersed phase conductivities based on Equation 1. (b) Electric circuit diagram derived from different electrical components as taken from cross section schematic inset. 
Figure 4a shows that the voltage across the droplet $V_{F}$ was higher at a lower conductivity. This trend agrees with experimental data of Figure 3, where the "Merge at Pillar" regime was observed only at a lower conductivity. Similarly, the voltage across the droplet also results in shifting of the "Inconsistent Merging at Pillar” regime, which gradually disappears at 10,200 $\mu$ S/cm. Although the higher conductivities result in a lower $V_{F}$, merging after the pillar was still observed as the overall electric field applied on the system still satisfies the minimum threshold voltage.

Finally, we demonstrated the proof-of-concept for on-demand size-based sorting of droplets using an expansion channel. Size-based sorting was previously demonstrated to separate droplets according to their contents ${ }^{35}$ or removal of by-products for the generation of monodispersed droplets. ${ }^{47}$ This approach reduces the need for further identification of droplets, eliminating human error and increasing accuracy. We modified the device design to include a second oil inlet to adjust spacing between droplets and the expansion channel after the electrodes, Figure 5a. The addition of the second oil inlet increased the distance between droplets, allowing well controlled droplet merging throughout the experiment. The additional inlet causes asymmetric breakup of the droplets. The expansion channel separates droplets according to their sizes due to the shear-gradient lift force and wall-effect lift force. ${ }^{35}$ Smaller droplets travel along the side of the walls along an equilibrium line arising due to lower wall-effect lift forces and exit through the waste outlets. ${ }^{1}$ Larger droplets remain in the middle stream along the middle equilibrium line due to the higher wall-effect lift force and subsequently collected through the middle outlet. If the AC electric field
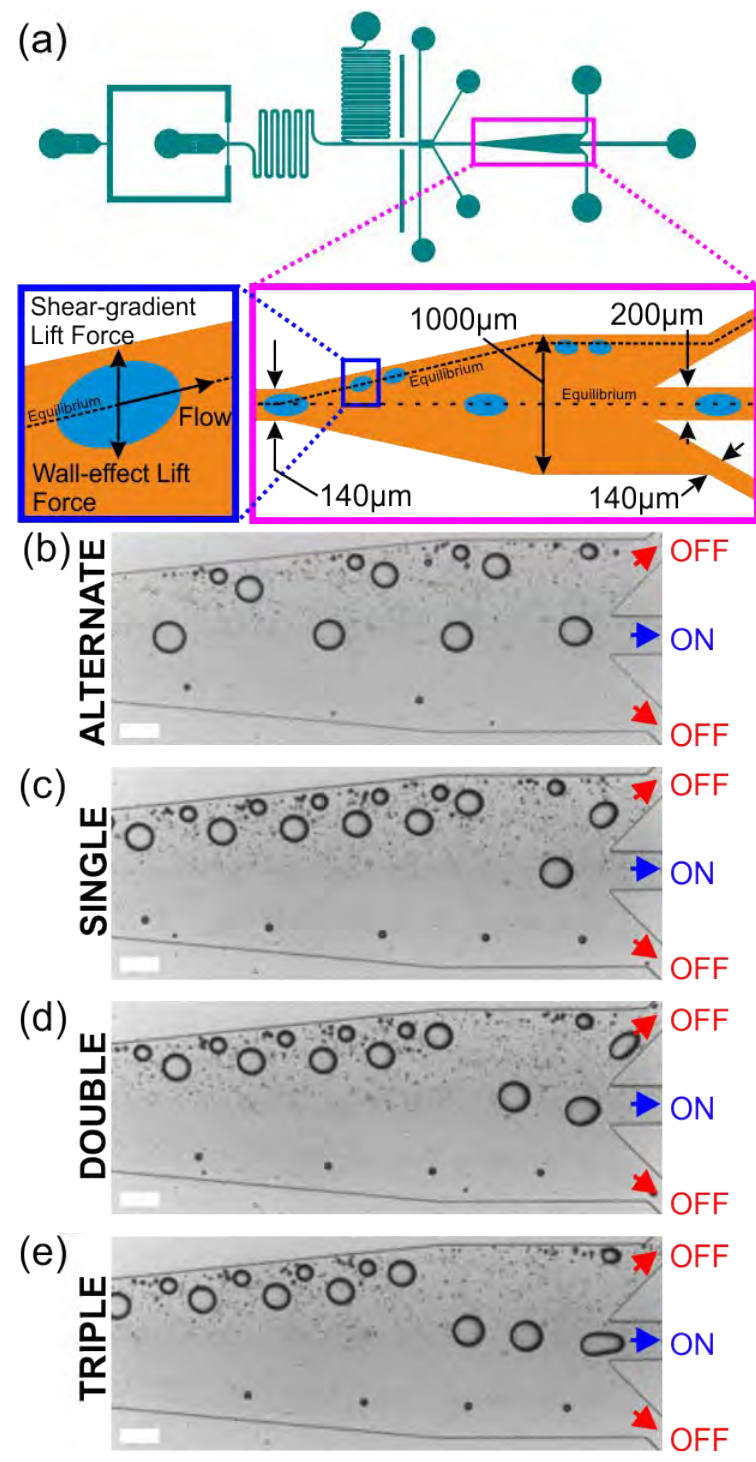

Figure 5. On-demand size based droplet sorting and generation: (a) Modified device with schematic of the expansion channel. An inset showing the forces influencing the path of the droplet flow is given. ${ }^{1}$ (b-e) On demand size-based droplet sorting where different electrical signals are applied to produce alternate, single, double and triple droplets. Red arrows show the flow path of separated droplets when AC electric field is OFF. Blue arrows show the path of merged droplets when AC electric field is ON. Scale bars depicts $200 \mu \mathrm{m}$. 
is turned off, small asymmetric droplets flow into the OFF outlets. With the AC field switched on, merged large droplets are accordingly collected from the middle ON outlet.

In this experiment, mineral oil with 1 wt $\%$ SPAN80 was introduced into both oil inlets at $500 \mu \mathrm{L} / \mathrm{hr}$ and $2000 \mu \mathrm{L} / \mathrm{hr}$. DI water with a conductivity of $0.67 \mu \mathrm{S} / \mathrm{cm}$ was used for the dispersed phase at a flow rate of $160 \mu \mathrm{L} / \mathrm{hr}$ to form droplets with sizes similar to the earlier experiments. Droplet frequency at the pillar was recorded to be approximately 28 droplets/second. The base AC signal used throughout the experiments is a sine wave at $10 \mathrm{kHz}$ and $156 \mathrm{~V}_{\mathrm{RMS}}$ (root-mean-square voltage). Applying a $14 \mathrm{~Hz}$ amplitude modulation on the AC signal resulted in alternate droplet merging. The merged droplets were successfully separated from the unmerged droplets, Figure 5b. We repeated the experiment with periodic bursts of the same sine waves in durations of 36, 72 and 107 milliseconds to achieve single, double and triple droplet generation, Figure 5c-e. The different configurations allow for accurate droplet dispensing, where users can control the volume of the dispersed phase according to the number of merged droplets. The merged droplets accordingly move out from the middle outlet. Users can subsequently introduce the next stage such as detection, reaction or drug dispensing ${ }^{48,49}$. Representative videos of the droplet sorting are given in Supporting Information S3.

As observed earlier, our system can effectively generate droplets of different volumes on demand. This multi-volume generation capability can be used for multi-volume digital polymerase chain reaction (MV-dPCR). ${ }^{50,51} \mathrm{MV}$-dPCR is a technique used for amplifying specific DNA sequences, where different volumes of samples are processed and analyzed simultaneously. The advantages of MV-dPCR are higher detection reproducibility and a wider dynamic range across different concentrations of samples equivalent to serial dilution measurements. ${ }^{52}$ By introducing the PCR mix and DNA template as the dispersed phase, individual PCR microreactors are formed. Splitting and coalescence of these microreactor droplets at the pillar can also be performed. This subsequently can be sorted passively with the use of the expansion channel. Without multiple parallel droplet generators, our device was able to dispense droplets of multiple volumes as demonstrated in Figure 5. Increasing the number of outlets in our device allows for sorting of 4 to 5 different droplet sizes, which can ultimately be used for such MV-dPCR analysis. Simultaneously, another potential application for this is in the dispensing of chemical reagents into droplets through the use of reagent loaded porous PDMS pillars. ${ }^{53}$ Through the activation of the electric field, droplets merging at the pillar enables the stored reagents within the pillar to come into contact with the droplet. Diffusion of the chemicals into the droplets would then occur allowing controlled release of chemicals.

\section{CONCLUSIONS}

We successfully demonstrated on-demand droplet splitting and merging using a perpendicular AC electric field. The concept employs a round micropillar placed in the middle of a microchannel, splitting the passing droplets. Electrodes placed across the pillar generates an AC electric field that induces polarization of the split daughter droplets. This process accordingly results in subsequent merging of the split daughter droplets due to dipole-dipole coalescence. The coalescence process depends on the magnitude and frequency of the AC electric field. Adjustment of the applied frequency $f$ and magnitude of the AC voltage $V_{A P P}$ results in three different regimes with two transitional regimes. The threshold voltage across different frequency values indicate the significant role of surfactant in the continuous phase. Subsequent adjustment of the conductivity $k$ of the dispersed phase shows a shift in the "Merging At Pillar" regime. We hypothesize that the voltage across the split droplets affects the transition of the merging regime due to increasing polarization. The circuit model of the electric system reveals that the voltage across the daughter droplets $V_{F}$ is higher at lower conductivities, agreeing well with experimental data.

We subsequently proceeded with size-based droplet sorting. An expansion microchannel separated unmerged droplets away from merged droplets that are collected from the middle outlet. We demonstrated the capabilities of alternate droplet merging and sorting as well as dispensing of single-droplet, double-droplet and triple-droplet volumes using pulsed AC voltage. We envision that the device concept reported here is of great value for applications such as controlled chemical release, drug dispensing or multi-volume digital polymerase chain reaction (MV-dPCR).

\section{AUTHOR INFORMATION}

\section{Corresponding Author}

Phone: +61-07373-53921.

E-mail: nam-trung.nguyen@griffith.edu.au. 


\section{ORCID}

Adrian J.T. Teo: 0000-0003-3779-6535

Say Hwa Tan: 0000-0002-9764-7868

Nam-Trung Nguyen: 0000-00003-3626-5361

\section{Notes}

The author declares no competing financial interest

\section{ACKNOWLEDGEMENT}

The authors acknowledge the Australian Research Council for funding support through the grant DE170100600. This work was performed in part at the Queensland node of the Australian National Fabrication Facility, a company established under the National Collaborative Research Infrastructure Strategy to provide nano- and micro-fabrication facilities for Australia's researchers.

\section{ASSOCIATED CONTENT}

\section{Supporting Information}

S1. Effects of Droplet Size on Coalescence

S2. Video of Different Merging Regimes Observed

S3. Video of Droplet Sorting

\section{REFERENCES}

(1) Di Carlo, D. Inertial microfluidics, Lab on a Chip 2009, 9, 3038-3046.

(2) Wang, J.; Teo, A. J. T.; Tan, S. H.; Evans, G. M.; Nguyen, N.-T.; Nguyen, A. V. Influence of Interfacial Gas Enrichment on Controlled Coalescence of Oil Droplets in Water in Microfluidics, Langmuir 2019, 35, 3615-3623.

(3) Fallah-Araghi, A.; Baret, J.-C.; Ryckelynck, M.; Griffiths, A. D. A completely in vitro ultrahigh-throughput droplet-based microfluidic screening system for protein engineering and directed evolution, Lab on a Chip 2012, 12, 882-891.

(4) Brouzes, E.; Medkova, M.; Savenelli, N.; Marran, D.; Twardowski, M.; Hutchison, J. B.; Rothberg, J. M.; Link, D. R.; Perrimon, N.; Samuels, M. L. Droplet microfluidic technology for single-cell high-throughput screening, Proceedings of the National Academy of Sciences 2009, 106, 1419514200.

(5) Krebs, T.; Schroen, C.; Boom, R. A microfluidic study of oil-water separation kinetics, Advances in Fluid Mechanics IX 2012, 74, 427-438.

(6) Xu, B.; Nguyen, N.-T.; Neng Wong, T. Droplet coalescence in microfluidic systems, Micro and Nanosystems 2011, 3, 131-136.

(7) Shen, F.; Li, Y.; Liu, Z.-M.; Cao, R.-T.; Wang, G.-R. Advances in Micro-Droplets Coalescence Using Microfluidics, Chinese Journal of Analytical Chemistry 2015, 43, 1942-1954.

(8) Liao, Y.; Lucas, D. A literature review on mechanisms and models for the coalescence process of fluid particles, Chemical Engineering Science 2010, 65, 2851-2864.

(9) Niu, X.; Gulati, S.; Edel, J. B. Pillar-induced droplet merging in microfluidic circuits, Lab on a chip 2008, 8, 1837-1841.

(10) Bremond, N.; Thiam, A. R.; Bibette, J. Decompressing Emulsion Droplets Favors Coalescence, Physical Review Letters $2008,100,024501$.

(11) Gu, H.; Duits, M. H.; Mugele, F. Droplets formation and merging in two-phase flow microfluidics, International journal of molecular sciences 2011, 12, 2572-2597.

(12) Yoon, D. H.; Jamshaid, A.; Ito, J.; Nakahara, A.; Tanaka, D.; Akitsu, T.; Sekiguchi, T.; Shoji, S. Active microdroplet merging by hydrodynamic flow control using a pneumatic actuator-assisted pillar structure, Lab on a Chip 2014, 14, 3050-3055.

(13) Sesen, M.; Alan, T.; Neild, A. Microfluidic on-demand droplet merging using surface acoustic waves, Lab on a Chip 2014, 14, 3325 -3333.

(14) Fidalgo, L. M.; Whyte, G.; Bratton, D.; Kaminski, C. F.; Abell, C.; Huck, W. T. From microdroplets to microfluidics: selective emulsion separation in microfluidic devices, Angewandte Chemie International Edition 2008, 47, 2042-2045.

(15) Chabert, M.; Dorfman, K. D.; Viovy, J. L. Droplet fusion by alternating current (AC) field electrocoalescence in microchannels, Electrophoresis 2005, 26, 3706-3715. 
(16) Wang, B.-B.; Wang, X.-D.; Wang, T.-H.; Yan, W.-M. Electrocoalescence behavior of two identical droplets with various droplet radii, Applied Thermal Engineering 2017, 111, 1464-1469.

(17) Eow, J. S.; Ghadiri, M.; Sharif, A. O.; Williams, T. J. Electrostatic enhancement of coalescence of water droplets in oil: a review of the current understanding, Chemical Engineering Journal 2001, 84, 173-192.

(18) Fritsch, R. J.; Krause, I. In Encyclopedia of Food Sciences and Nutrition (Second Edition), Caballero, B., Ed.; Academic Press: Oxford, 2003, pp 2055-2062.

(19) Im, D. J.; Noh, J.; Moon, D.; Kang, I. S. Electrophoresis of a Charged Droplet in a Dielectric Liquid for Droplet Actuation, Analytical Chemistry 2011, 83, 5168-5174.

(20) Makino, K.; Ohshima, H. Electrophoretic Mobility of a Colloidal Particle with Constant Surface Charge Density, Langmuir 2010, 26, 1801618019.

(21) Xi, H.-D.; Guo, W.; Leniart, M.; Chong, Z. Z.; Tan, S. H. AC electric field induced droplet deformation in a microfluidic T-junction, Lab on a Chip 2016, 16, 2982-2986.

(22) Stone, H. A.; Lister, J. R.; Brenner, M. P. Drops with conical ends in electric and magnetic fields, Proceedings of the Royal Society of London. Series A: Mathematical, Physical and Engineering Sciences 1999, 455, 329-347.

(23) Chen, X.; Song, Y.; Li, D.; Hu, G. Deformation and interaction of droplet pairs in a microchannel under ac electric fields, Physical Review Applied 2015, 4, 024005.

(24) Peng, Y.; Liu, T.; Gong, H.; Zhang, X. Review of the dynamics of coalescence and demulsification by high-voltage pulsed electric fields, International Journal of Chemical Engineering 2016, 2016.

(25) Lukyanets, A.; Kavehpour, H. Effect of electric fields on the rest time of coalescing drops, Applied Physics Letters $2008,93,194101$.

(26) Wang, W.; Yang, C.; Li, C. M. On-demand microfluidic droplet trapping and fusion for on-chip static droplet assays, Lab on a Chip 2009, 9, 1504-1506.

(27) Lee, H.-J.; Chin, B. D.; Yang, S.-M.; Park, O. O. Surfactant effect on the stability and electrorheological properties of polyaniline particle suspension, Journal of colloid and interface science 1998, 206, 424-438.

(28) Mannoor, M.; Kang, S.; Suh, Y. K. Molecular Dynamics Simulation of Aggregates in the Dodecane/span80 System and Their Behaviour in an Electric Field, Advances in Condensed Matter Physics 2015, 2015, 9.

(29) Wang, B.-B.; Wang, X.-D.; Yan, W.-M.; Wang, T.-H. Molecular Dynamics Simulations on Coalescence and Non-coalescence of Conducting Droplets, Langmuir 2015, 31, 7457-7462.

(30) Niu, X.; Gielen, F.; deMello, A. J.; Edel, J. B. Electro-Coalescence of Digitally Controlled Droplets, Analytical Chemistry 2009, 81, 7321-7325.

(31) Baygents, J. C.; Rivette, N.; Stone, H. A. Electrohydrodynamic deformation and interaction of drop pairs, Journal of Fluid Mechanics 1998, 368, 359-375.

(32) Li, Q.; Chai, Z.; Shi, B.; Liang, H. Deformation and breakup of a liquid droplet past a solid circular cylinder: A lattice Boltzmann study, Physical Review E 2014, 90, 043015.

(33) Chung, C.; Lee, M.; Char, K.; Ahn, K. H.; Lee, S. J. Droplet dynamics passing through obstructions in confined microchannel flow, Microfluidics and Nanofluidics 2010, 9, 1151-1163.

(34) Xi, H.-D.; Zheng, H.; Guo, W.; Ganan-Calvo, A. M.; Ai, Y.; Tsao, C.-W.; Zhou, J.; Li, W.; Huang, Y.; Nguyen, N.-T.; Tan, S. H. Active droplet sorting in microfluidics: a review, Lab on a Chip 2017, 17, 751-771.

(35) Li, M.; van Zee, M.; Goda, K.; Di Carlo, D. Size-based sorting of hydrogel droplets using inertial microfluidics, Lab on a Chip 2018, 18, 25752582.

(36) Ma, Z.; Teo, A. J. T.; Tan, S. H.; Ai, Y.; Nguyen, N.-T. Self-Aligned Interdigitated Transducers for Acoustofluidics, Micromachines 2016, 7 , 216.

(37) Guo, W.; Teo, A. J. T.; Gañán-Calvo, A. M.; Song, C.; Nguyen, N.-T.; Xi, H.-D.; Tan, S. H. Pressure-Driven Filling of Closed-End Microchannel: Realization of Comb-Shaped Transducers for Acoustofluidics, Physical Review Applied 2018, 10, 054045.

(38) Gañán-Calvo, A. M.; Guo, W.; Xi, H.-D.; Teo, A. J.; Nguyen, N.-T.; Tan, S. H. Pressure-driven filling of liquid metal in closed-end microchannels, Physical Review E 2018, 98, 032602.

(39) Teo, A. J. T.; Li, K.-H. H.; Nguyen, N.-T.; Guo, W.; Heere, N.; Xi, H.-D.; Tsao, C.-W.; Li, W.; Tan, S. H. Negative Pressure Induced Droplet Generation in a Microfluidic Flow-Focusing Device, Analytical Chemistry 2017.

(40) Mandal, S.; Chaudhury, K.; Chakraborty, S. Transient dynamics of confined liquid drops in a uniform electric field, Physical Review E 2014, 89, 053020 . 
(41) Priest, C.; Herminghaus, S.; Seemann, R. Controlled electrocoalescence in microfluidics: Targeting a single lamella, Applied Physics Letters 2006, 89, 134101.

(42) Baret, J.-C.; Miller, O. J.; Taly, V.; Ryckelynck, M.; El-Harrak, A.; Frenz, L.; Rick, C.; Samuels, M. L.; Hutchison, J. B.; Agresti, J. J.; Link, D. R.; Weitz, D. A.; Griffiths, A. D. Fluorescence-activated droplet sorting (FADS): efficient microfluidic cell sorting based on enzymatic activity, Lab on a Chip 2009, 9, 1850-1858.

(43) Sciambi, A.; Abate, A. R. Accurate microfluidic sorting of droplets at $30 \mathrm{kHz}$, Lab on a Chip 2015, 15, 47-51.

(44) Bashir, S.; Solvas, X. C. i.; Bashir, M.; Rees, J. M.; Zimmerman, W. B. J. Dynamic wetting in microfluidic droplet formation, BioChip Journal 2014, 8, 122-128.

(45) Abou-Nemeh, I.; Bart, H. J. Microstructures in the System Water/D2EHPA/Span-80/n-Dodecane, Langmuir 1998, 14, 4451-4459.

(46) Drews, A. M.; Lee, H.-Y.; Bishop, K. J. Ratcheted electrophoresis for rapid particle transport, Lab on a Chip 2013, 13, 4295-4298.

(47) Yang, C.-H.; Lin, Y.-S.; Huang, K.-S.; Huang, Y.-C.; Wang, E.-C.; Jhong, J.-Y.; Kuo, C.-Y. Microfluidic emulsification and sorting assisted preparation of monodisperse chitosan microparticles, Lab on a Chip 2009, 9, 145-150.

(48) Wang, B.; Ni, J.; Litvin, Y.; Pfaff, D. W.; Lin, Q. A microfluidic approach to pulsatile delivery of drugs for neurobiological studies, Journal of Microelectromechanical Systems 2011, 21, 53-61.

(49) Cole, R. H.; Tang, S.-Y.; Siltanen, C. A.; Shahi, P.; Zhang, J. Q.; Poust, S.; Gartner, Z. J.; Abate, A. R. Printed droplet microfluidics for on demand dispensing of picoliter droplets and cells, Proceedings of the National Academy of Sciences 2017, 114, 8728-8733.

(50) Zeng, Y.; Shin, M.; Wang, T. Programmable active droplet generation enabled by integrated pneumatic micropumps, Lab on a Chip 2013, 13, 267-273.

(51) Shen, F.; Sun, B.; Kreutz, J. E.; Davydova, E. K.; Du, W.; Reddy, P. L.; Joseph, L. J.; Ismagilov, R. F. Multiplexed Quantification of Nucleic Acids with Large Dynamic Range Using Multivolume Digital RT-PCR on a Rotational SlipChip Tested with HIV and Hepatitis C Viral Load, Journal of the American Chemical Society 2011, 133, 17705-17712.

(52) Zhu, P.; Wang, L. Passive and active droplet generation with microfluidics: a review, Lab on a Chip 2017.

(53) Thurgood, P.; Baratchi, S.; Szydzik, C.; Mitchell, A.; Khoshmanesh, K. Porous PDMS structures for the storage and release of aqueous solutions into fluidic environments, Lab on a Chip 2017, 17, 2517-2527.

\section{Table of Contents artwork}

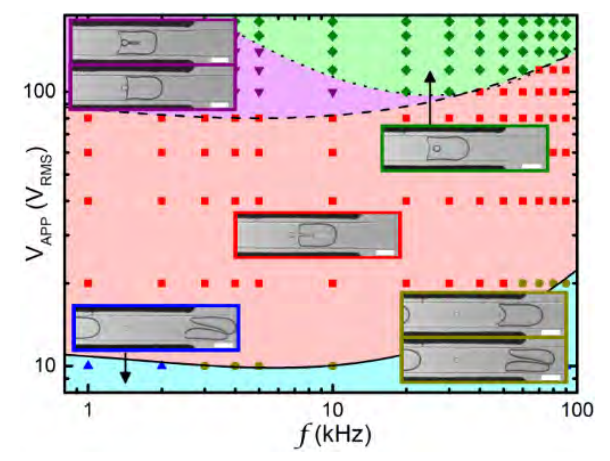

